\title{
Effects of algal diets and starvation on growth, survival and fatty acid composition of Solen marginatus (Bivalvia: Solenidae) larvae
}

\author{
FIZ DA COSTA, SUSANA NÓVOA, JUSTA OJEA and DOROTEA MARTÍNEZ-PATIÑO \\ Centro de Investigacións Mariñas, Consellería do Mar, Xunta de Galicia, Peirao de Porcillán s/n, 27700, Ribadeo, \\ Lugo, Spain. E-mail: dacosta.fiz@ cimacoron.org
}

\begin{abstract}
SUMMARY: The aim of this study was to investigate whether it is necessary to feed Solen marginatus (Pennánt, 1777) larvae externally and the evolution of fatty acids in the neutral and polar lipids during larval development in starved larvae and larvae fed on two different microalgal diets. Larvae were subjected to three different treatments: 1.10 equivalent cells (Isochrysis galbana, Pavlova lutheri and Chaetoceros calcitrans) plus 20 equivalent cells of Tetraselmis suecica; 2. 80 equivalent cells of I. galbana; and 3. starvation during eight days, and then individuals were re-fed on diet 1 . The best results for growth were observed in larvae fed on diet 1 . Starved larvae reached the best survival rate at day $8(66 \%)$. However, three days after re-feeding all larvae died, suggesting that the "point of no return" was exceeded. In spite of the large size of $S$. marginatus eggs and the great amount of stored reserves, the larvae need to feed on microalgae to undergo metamorphosis. Non-methyle-interrupted dienoic fatty acids and their precursors 16:1n-7 and 18:1n-9 are of great importance in starved larvae. Saturated fatty acids, especially 16:0, fuel larval development. A certain degree of bioconversion of 18:2n-6 to 20:4n-6 was observed in S. marginatus larvae.
\end{abstract}

Keywords: Solen marginatus, razor clam, larvae, diets, starvation.

RESUMEN: EFeCtos DE LAS DIETAS ALGALES Y EL AYUNO EN EL CRECIMIENTO, SUPERVIVENCIA Y COMPOSICIÓN DE ÁCIDOS GRASos de las larvas de Solen marginatus (Bivalvia: Solenidae). - El objetivo de este estudio es investigar la necesidad de alimentación externa de las larvas de Solen marginatus (Pennánt, 1777) y la evolución de los ácidos grasos en los lípidos neutros y polares durante el desarrollo larvario en ayuno y usando dos dietas microalgales. Las larvas se sometieron a tres tratamientos distintos: 1.10 células equivalentes Isochrysis galbana, Pavlova lutheri y Chaetoceros calcitrans) más 20 células equivalentes de Tetraselmis suecica; 2.80 células equivalentes de I. galbana; y 3. Ayuno durante ocho días, y después los individuos se realimentaron con la dieta 1 . Los mejores resultados en crecimiento se observaron en las larvas alimentadas con la dieta 1 . Las larvas que ayunaron alcanzaron la mejor supervivencia en el día 8 (66\%). Sin embargo, tres días después de volver a alimentarlas murieron todas, sugiriendo que el "punto de no retorno" se sobrepasó. A pesar del gran tamaño de los huevos de $S$. marginatus y del gran acúmulo de reservas sus larvas necesitan alimentarse externamente para llevar a cabo la metamorfosis. Los ácidos grasos dienoicos no-metilen-interrumpidos y sus precursores 16:1n-7 y 18:1n-9 son de gran importancia para las larvas en ayuno. Los ácidos grasos saturados, especialmente el 16:0, suministran energía para el desarrollo larvario. Se ha observado cierta bioconversión de 18:2n-6 en 20:4n-6 en las larvas de S. marginatus.

Palabras clave: Solen marginatus, longueirón, larva, dietas, ayuno.

\section{INTRODUCTION}

The razor clam Solen marginatus (Pennánt, 1777) is the most abundant species of Solenidae in Spain. $S$. marginatus lives buried in sand and/or muddy sand in low intertidal and subtidal zones from Norway to the Mediterranean, Black Sea and West Africa. Razor clams are harvested mainly in Europe, Africa, Asia and America, and $S$. marginatus is a valuable shellfish resource in Spain, Portugal, Italy, Tunisia, Morocco and Greece. Wild populations of this species are threatened due to over-fishing, environmental pollution associ- 
ated with the estuarine areas that the species inhabits, and deficient management of the natural beds. Hatchery production has accordingly gained importance in order to replenish natural beds and to reinforce recruitment. Therefore, hatchery production is of the utmost importance and the first investigations into juvenile production in land-based facilities have been recently carried out (da Costa and Martinez-Patiño 2009). Diets used in razor clam hatcheries have generally been adapted from those used for other bivalves with more established culture practices, yet the performance of algal diets is often highly species- and stage-specific. Hence, optimizing the species composition of microalgal diets for a specific animal is of vital importance. However, there is currently little information on the nutritional requirements of $S$. marginatus larvae and no study has been previously reported on the fatty acid composition of $S$. marginatus larvae exposed to different diets and starvation.

S. marginatus eggs are rich in stored reserves given their large size $(150 \mu \mathrm{m})$. The chorionic envelope and the lipidic droplet provide energy for embryonic development (da Costa et al. 2011), which leads to the fast larval development exhibited in this species, which only lasts 8 days. Ockelmann (1965) found that the lecithotrophic mode of development occurs when the diameter of the yolk-mass of ripe eggs is larger than $90 \mu \mathrm{m}$, and these species all have a very short pelagic life. Owing to a richer supply of nutrient matter from the egg, these larvae have also become independent of planktonic food. Thus, the large egg of $S$. marginatus may have lecithotrophic larval development in which food acquisition from plankton is not necessary. Despite this supposition, larval rearing has been successfully carried out by supplying a mixed diet (da Costa and MartinezPatiño 2009). In the present work we tested starvation to ascertain if $S$. marginatus larvae are able to settle under phytoplankton deprivation. In starvation experiments the term "point-of-no-return" or PNR is commonly used and denotes the threshold above which larvae can no longer metamorphose even if food was provided again.

Nutrition is one of the dominant factors influencing larval bivalve growth and survival, and has been extensively reviewed in Marshall et al. (2010). The criteria for selecting a suitable algal diet for bivalve larvae must be based on form, mobility, size, toxicity and the ability of the larvae to trap, ingest, digest and assimilate the alga. Moreover, the food value is determined mostly based on the biochemical composition (lipid, carbohydrate and protein). Essential fatty acids (EFAs), particularly omega-3 fatty acids, eicosapentaenoic acid (20:5n-3, EPA) and docosahexaenoic acid (22:6n-3, DHA), are important for growth and development (Langdon and Waldock 1981) because they are major membrane components (Hendriks et al. 2003) and possible modulators of the membrane function (Palacios et al. 2005). Bivalves have a very limited capacity for synthesizing EFAs and it is inadequate for meeting their nutritional requirements; therefore, they must be supplied exogenously (Laing et al. 1990, Chu and Greaves 1991).
It is widely accepted that mixed diets meet better nutritional requirements of bivalve larvae. Isochrysis galbana is a food used worldwide for cultured suspension-feeding bivalves (Webb and Chu 1982) and in the present study it was delivered to razor clams as a standard food particle, and compared with a mixed diet. The main aim of the present work was to test whether it is necessary to externally feed $S$. marginatus larvae and also the dynamics of fatty acids in the neutral and polar lipids during starvation and with feeding on two phytoplankton diets.

\section{MATERIALS AND METHODS}

\section{Larval culture}

Adult $S$. marginatus (>80 $\mathrm{mm}$ shell length) were collected from a natural bed in an intertidal area of Redondela, in Ría de Vigo (42 ${ }^{\circ} 29^{\prime} \mathrm{N}$; 08 $04^{\circ}$ 'W) (Galicia, NW Spain). Broodstocks were arranged in 200-L rectangular fibreglass tanks at $19 \pm 1{ }^{\circ} \mathrm{C}$ in an open circuit buried in a $30 \mathrm{~cm}$ layer of sand for 2 weeks. The individuals were fed daily with a mixed culture of Tetraselmis suecica, Isochrysis galbana, Pavlova lutheri, Chaetoceros calcitrans, Phaeodactylum tricornutum and Skeletonema marinoï in equal proportions, representing a ration of 5.5\% of dry meat weight in dry algal weight per day $\left(3 \times 10^{9}\right.$ cells/razor clam/day). Fifty individuals were placed into $200 \mathrm{~L}$ tanks and held on row bars tied individually with rubber bands for spawning induction. Razor clams were subjected to thermal shock, with temperatures up to 25$27^{\circ} \mathrm{C}$ for $1 \mathrm{~h}$, decreasing to $10-12^{\circ} \mathrm{C}$ for $30 \mathrm{~min}$. A total of 3-4 cycles were performed. An additional stimulus was provided by adding stripped gametes and microalgae. The specimens responding to the stimulus were separated into individual $1 \mathrm{~L}$ test tubes for the release of sperm or eggs, thus avoiding polyspermy. A mixture of sperm from 3 to 4 males was used for fertilization at a ratio of $2 \mathrm{ml}$ of sperm per litre of egg suspension (Helm et al. 2004). Fertilization was conducted in a 5-1 flask. After fertilization, the eggs were sieved through a 60$\mathrm{lm}$ screen to eliminate excess sperm. Then, the embryos were transferred to $150-\mathrm{L}$ larval culture tanks. The temperature was set at $19 \pm 1^{\circ} \mathrm{C}$ and water was changed every second day using $1 \mu \mathrm{m}$ sand-filtered, UV-sterilized seawater. No food was provided during embryo incubation. The experiment started on day 1 and larvae were split into six batches for duplicate exposure to three nutritional regimes: 1: A mixed diet consisting of 10 cells $\mu \mathrm{L}^{-1}$ of I. galbana, P. lutheri and C. calcitrans, plus 20 cells $\mu \mathrm{L}^{-1}$ of $T$. suecica; 2 : A single diet consisting of 80 cells $\mu \mathrm{L}^{-1}$ of I. galbana; $3: 8$ days of starvation (until settlement occurred in treatments 1 and 2), and thereafter razor clam larvae were re-fed on diet 1 . Larval density was set at 5 individuals $\mathrm{mL}^{-1}$ at the beginning of the experiment. Larvae were collected with nytex screens and the shell length was measured on 100 individuals per tank using a binocular microscope connected to an image analyzer to determine the growth pattern. 
TABle 1. - Growth and survival of fed or unfed Solen marginatus larvae on day 8.

\begin{tabular}{lccc}
\hline & $\begin{array}{c}\text { Length } \\
(\mu \mathrm{m})\end{array}$ & $\begin{array}{c}\text { Growth rate } \\
\left(\mu \mathrm{m} \mathrm{day}^{-1}\right)\end{array}$ & Survival (\%) \\
\hline Diet 1- Mixture & $287.51 \pm 0.18^{\mathrm{a}}$ & $19.16 \pm 0.02$ & $48.96 \pm 8.22^{\mathrm{a}}$ \\
Diet 2- . galbana & $266.30 \pm 2.87^{\mathrm{a}}$ & $16.13 \pm 0.41$ & $28.86 \pm 2.68^{\mathrm{b}}$ \\
Diet 3- Starvation & $180.82 \pm 2.18^{\mathrm{b}}$ & $3.92 \pm 0.31$ & $66.36 \pm 1.49^{\mathrm{c}}$ \\
\hline
\end{tabular}

\section{Fatty acid analysis}

Samples of 20,000 larvae (day 1 and 8) per tank and four samples of diets were filtered and crushed on Whatman GF/C filters, washed with $3 \%$ ammonium formate and stored in a $\mathrm{CHCl}_{3}-\mathrm{MeOH}$ mixture $(2: 1$, $\mathrm{v} / \mathrm{v})$ at $-20^{\circ} \mathrm{C}$. The microalgal mixture was sampled at the exponential phase of culture (7 days). The neutral and polar lipids were separated by micro-column liquid chromatography as described by Marty et al. (1992). Total lipids were evaporated to dryness and dissolved three times using $500 \mu \mathrm{L}$ of chloroform/methanol (98:2). Neutral and polar lipids were separated on a silica gel $6 \%(\mathrm{w} / \mathrm{w})$ hydrated microcolumn $(30 \times 5 \mathrm{~mm})$ using chloroform/methanol (98:2) and methanol successively as eluting solvents. The fractions were collected under nitrogen in a screw-capped flask containing a known amount of 23:0 as internal standard for quantitative determinations. Fatty acids from the neutral and polar lipid fractions were transesterified with $10 \%(\mathrm{w} / \mathrm{w}) \mathrm{BF}_{3}$ in methanol (Metcalfe and Schmitz 1961) for $10 \mathrm{~min}$ at $100^{\circ} \mathrm{C}$. After cooling, fatty acid methyl esters were extracted with hexane. The organic phase was evaporated under nitrogen and dissolved in hexane for purification by HPLC (Soudant et al. 1995). The fatty acid composition and quantification of neutral and polar lipids were determined using gas chromatography (Variant, CP-3800), equipped with a fused silica capillary column (JW SCIEN, $30 \mathrm{~m}$ length, $0.25 \mathrm{~mm}$ i.d., $0.25 \mu \mathrm{m}$ film thickness), with a cool on-column injector at $63^{\circ} \mathrm{C}$. The carrier gas was $\mathrm{H}_{2}$, at a initial pressure of $80 \mathrm{kPa}$. The oven was programmed to stay at an initial temperature of $60^{\circ} \mathrm{C}$ for $2 \mathrm{~min}$, increase from 60 to $160^{\circ} \mathrm{C}$ at a rate $50^{\circ} \mathrm{C} / \mathrm{min}$, stay there for $2 \mathrm{~min}$, then increase from 160 to $170^{\circ} \mathrm{C}$ at $1.5^{\circ} \mathrm{C} / \mathrm{min}$, then from 170 to $185^{\circ} \mathrm{C}$ at $2^{\circ} \mathrm{C} /$ min, then from $185-240^{\circ} \mathrm{C}$ at $3^{\circ} \mathrm{C} / \mathrm{min}$, and finally remain at $240^{\circ} \mathrm{C}$ for $10 \mathrm{~min}$. The detector temperature was $250^{\circ} \mathrm{C}$. Fatty acids were identified by comparing their retention times with those of standards. A response factor was calculated for each fatty acid in order to perform quantitative analyses.

\section{Statistical analyses}

Group normality (shell length) was initially evaluated using an Kolmogorov-Smirnov test and then one-way ANOVA for significant differences between groups. The homogeneity of variances was checked by means of a Barlett test. When necessary, post hoc analyses with Tukey's multiple range test were applied. Percentage data were arcsine-transformed to
TABLE 2. - Fatty acid composition of total lipids of the two diets tested (expressed as mean \pm S.D. percentage).

\begin{tabular}{|c|c|c|}
\hline & Diet 1- Mixture & Diet $2-I$ galbana \\
\hline 14:0 & $19.95 \pm 0.22^{\mathrm{a}}$ & $12.97 \pm 1.21^{\mathrm{b}}$ \\
\hline 16:0 & $7.43 \pm 0.02^{\mathrm{a}}$ & $9.12 \pm 0.04^{\mathrm{b}}$ \\
\hline 18:0 & $0.50 \pm 0.01^{\mathrm{a}}$ & $8.78 \pm 0.10^{\mathrm{b}}$ \\
\hline 20:0 & $0.00 \pm 0.00^{\mathrm{a}}$ & $2.72 \pm 0.04^{\mathrm{b}}$ \\
\hline $22: 0$ & $0.08 \pm 0.00^{\mathrm{a}}$ & $0.11 \pm 0.00^{\mathrm{a}}$ \\
\hline 24:0 & $0.18 \pm 0.00^{\mathrm{a}}$ & $0.14 \pm 0.00^{\mathrm{a}}$ \\
\hline $16: 1 n-9$ & $0.22 \pm 0.10^{\mathrm{a}}$ & $0.30 \pm 0.00^{\mathrm{a}}$ \\
\hline $16: 1 n-7$ & $5.11 \pm 0.08^{\mathrm{a}}$ & $5.63 \pm 0.02^{\mathrm{a}}$ \\
\hline $18: 1 n-11$ & $0.13 \pm 0.07^{\mathrm{a}}$ & $0.19 \pm 0.00^{\mathrm{a}}$ \\
\hline $18: 1 n-9$ & $10.99 \pm 0.17^{\mathrm{a}}$ & $1.80 \pm 0.03^{\mathrm{b}}$ \\
\hline $18: 1 n-7$ & $2.11 \pm 0.04^{\mathrm{a}}$ & $0.02 \pm 0.00^{\mathrm{b}}$ \\
\hline $20: 1 n-11$ & $0.00 \pm 0.00^{\mathrm{a}}$ & $0.00 \pm 0.00^{\mathrm{a}}$ \\
\hline $20: 1 n-9$ & $0.00 \pm 0.00^{\mathrm{a}}$ & $0.00 \pm 0.00^{\mathrm{a}}$ \\
\hline $20: 1 \mathrm{n}-7$ & $0.05 \pm 0.08^{\mathrm{a}}$ & $0.00 \pm 0.00^{\mathrm{b}}$ \\
\hline $16: 3 n-6$ & $0.00 \pm 0.00^{\mathrm{a}}$ & $0.00 \pm 0.00^{\mathrm{a}}$ \\
\hline $16: 3 n-4$ & $0.00 \pm 0.00^{\mathrm{a}}$ & $0.21 \pm 0.01^{\mathrm{b}}$ \\
\hline $16: 4 n-3$ & $0.51 \pm 0.01^{\mathrm{a}}$ & $0.00 \pm 0.00^{\mathrm{b}}$ \\
\hline $18: 2 n-6$ & $11.03 \pm 0.09^{\mathrm{a}}$ & $7.39 \pm 0.12^{b}$ \\
\hline $18: 2 n-4$ & $0.27 \pm 0.01^{\mathrm{a}}$ & $0.00 \pm 0.00^{\mathrm{b}}$ \\
\hline $18: 3 n-6$ & $1.39 \pm 0.04^{\mathrm{a}}$ & $0.43 \pm 0.01^{\mathrm{b}}$ \\
\hline $18: 3 n-3$ & $9.75 \pm 0.17^{\mathrm{a}}$ & $15.29 \pm 0.40^{\mathrm{b}}$ \\
\hline $18: 4 n-3$ & $9.51 \pm 0.08^{\mathrm{a}}$ & $18.33 \pm 0.39^{\mathrm{b}}$ \\
\hline $20: 2 \mathrm{i}$ & $0.00 \pm 0.00^{\mathrm{a}}$ & $0.00 \pm 0.00^{\mathrm{a}}$ \\
\hline $20: 2 \mathrm{j}$ & $0.00 \pm 0.00^{\mathrm{a}}$ & $0.00 \pm 0.00^{\mathrm{a}}$ \\
\hline $20: 2 n-6$ & $0.00 \pm 0.00^{\mathrm{a}}$ & $0.03 \pm 0.00^{\mathrm{b}}$ \\
\hline $20: 3 n-6$ & $0.08 \pm 0.01^{\mathrm{a}}$ & $0.03 \pm 0.00^{\mathrm{b}}$ \\
\hline $20: 4 n-6$ & $1.22 \pm 0.04^{\mathrm{a}}$ & $0.17 \pm 0.00^{\mathrm{b}}$ \\
\hline $20: 4 n-3$ & $0.04 \pm 0.00^{\mathrm{a}}$ & $0.01 \pm 0.01^{\mathrm{b}}$ \\
\hline $20: 5 n-3$ & $4.88 \pm 0.21^{\mathrm{a}}$ & $0.49 \pm 0.02^{\mathrm{b}}$ \\
\hline $21: 4 n-6$ & $0.25 \pm 0.02^{\mathrm{a}}$ & $0.00 \pm 0.00^{\mathrm{b}}$ \\
\hline $21: 5 n-3$ & $0.10 \pm 0.01^{\mathrm{a}}$ & $0.15 \pm 0.00^{\mathrm{a}}$ \\
\hline $22: 2 \mathrm{i}$ & $0.00 \pm 0.00^{\mathrm{a}}$ & $0.00 \pm 0.00^{\mathrm{a}}$ \\
\hline $22: 2 \mathrm{j}$ & $0.00 \pm 0.00^{\mathrm{a}}$ & $0.00 \pm 0.00$ \\
\hline $22: 4 n-6$ & $0.00 \pm 0.00^{\mathrm{a}}$ & $0.06 \pm 0.00^{\mathrm{b}}$ \\
\hline $22: 5 n-6$ & $1.20 \pm 0.01^{\mathrm{a}}$ & $1.66 \pm 0.00^{\mathrm{b}}$ \\
\hline $22: 5 n-3$ & $0.14 \pm 0.04^{\mathrm{a}}$ & $0.07 \pm 0.01^{\mathrm{a}}$ \\
\hline $22: 6 n-3$ & $6.83 \pm 0.12^{\mathrm{a}}$ & $10.19 \pm 0.26^{\mathrm{b}}$ \\
\hline$\sum$ SAFA & $28.40 \pm 0.28^{\mathrm{a}}$ & $34.19 \pm 1.05^{\mathrm{b}}$ \\
\hline$\sum$ MUFA & $20.07 \pm 0.05^{\mathrm{a}}$ & $8.88 \pm 0.03^{b}$ \\
\hline$\sum \mathrm{n}-9$ & $11.21 \pm 0.08^{\mathrm{a}}$ & $2.24 \pm 0.03^{\mathrm{b}}$ \\
\hline$\sum n-7$ & $8.36 \pm 0.16^{\mathrm{a}}$ & $6.34 \pm 0.03^{\mathrm{a}}$ \\
\hline$\sum$ PUFA & $49.57 \pm 0.32^{\mathrm{a}}$ & $55.69 \pm 1.16^{\mathrm{b}}$ \\
\hline$\sum n-6$ & $13.96 \pm 0.03^{\mathrm{a}}$ & $10.76 \pm 0.14^{b}$ \\
\hline$\sum n-3$ & $31.83 \pm 0.28^{\mathrm{a}}$ & $44.53 \pm 1.05^{\mathrm{b}}$ \\
\hline$\sum$ NMID & $0.00 \pm 0.00^{\mathrm{a}}$ & $0.00 \pm 0.00^{\mathrm{a}}$ \\
\hline n-3/n-6 & $2.28 \pm 0.02^{\mathrm{a}}$ & $4.14 \pm 0.05^{\mathrm{b}}$ \\
\hline $22: 6 / 20: 5$ & $1.40 \pm 0.04^{\mathrm{a}}$ & $20.60 \pm 0.14^{\mathrm{b}}$ \\
\hline $22: 5 / 20: 4$ & $0.98 \pm 0.02^{\mathrm{a}}$ & $9.98 \pm 0.01^{\mathrm{b}}$ \\
\hline $20: 5 / 20: 4$ & $4.00 \pm 0.11^{\mathrm{a}}$ & $2.88 \pm 0.18^{b}$ \\
\hline
\end{tabular}

Mean values within the same columns with different superscript letters are significantly different $(P<0.05)$.

normalize variance (Sokal and Rohlf 1995). Comparison of survival was performed using a Kruskal-Wallis non-parametric test followed by a pair wise U-MannWhitney test. Differences were considered to be statistically significant when $\mathrm{P}<0.05$.

\section{RESULTS}

\section{Growth and survival}

Larvae fed on a mixed diet (diet 1) exhibited a faster growth rate and longer length at settlement (day 8) than those fed with the I. galbana diet (diet 2) $(P<0.05)$ (Table 1). Starved larvae exhibited poor growth during 
TABLE 3. - Fatty acid composition of total neutral lipids of S. marginatus larvae (expressed as mean \pm S.D. of ng individual ${ }^{-1}$ ).

\begin{tabular}{|c|c|c|c|c|}
\hline & Day 1 & Mixture-Day 8 & I. galbana-Day 8 & Starvation-Day 8 \\
\hline 14:0 & $1.09 \pm 0.17^{\mathrm{a}}$ & $1.95 \pm 0.57^{b}$ & $2.43 \pm 0.61^{\mathrm{b}}$ & $0.15 \pm 0.03^{c}$ \\
\hline $16: 0$ & $3.57 \pm 0.27^{\mathrm{a}}$ & $2.83 \pm 0.77^{\mathrm{a}}$ & $3.28 \pm 0.79^{\mathrm{a}}$ & $0.58 \pm 0.10^{\mathrm{b}}$ \\
\hline 18:0 & $0.81 \pm 0.04^{\mathrm{a}}$ & $0.74 \pm 0.21^{\mathrm{a}}$ & $0.86 \pm 0.20^{\mathrm{a}}$ & $0.23 \pm 0.08^{\mathrm{b}}$ \\
\hline $16: 1 n-9$ & $0.06 \pm 0.05^{\mathrm{a}}$ & $0.06 \pm 0.03^{\mathrm{a}}$ & $0.09 \pm 0.02^{\mathrm{a}}$ & $0.01 \pm 0.00^{\mathrm{b}}$ \\
\hline $16: 1 n-7$ & $1.60 \pm 0.14^{\mathrm{a}}$ & $1.16 \pm 0.40^{\mathrm{a}}$ & $1.33 \pm 0.34^{\mathrm{a}}$ & $0.18 \pm 0.01^{\mathrm{b}}$ \\
\hline $18: 1 n-11$ & $0.00 \pm 0.00^{\mathrm{a}}$ & $0.06 \pm 0.05^{\mathrm{b}}$ & $0.00 \pm 0.00^{\mathrm{a}}$ & $0.00 \pm 0.00^{\mathrm{a}}$ \\
\hline $18: 1 n-9$ & $1.40 \pm 0.10^{\mathrm{a}}$ & $1.14 \pm 0.44^{\mathrm{a}}$ & $2.47 \pm 0.72^{b}$ & $0.29 \pm 0.04^{c}$ \\
\hline $18: 1 n-7$ & $0.60 \pm 0.05^{\mathrm{a}}$ & $0.59 \pm 0.22^{\mathrm{a}}$ & $0.68 \pm 0.19^{\mathrm{a}}$ & $0.10 \pm 0.01^{\mathrm{b}}$ \\
\hline $20: 1 n-11$ & $0.15 \pm 0.01^{\mathrm{a}}$ & $0.10 \pm 0.08^{\mathrm{a}}$ & $0.07 \pm 0.04^{\mathrm{b}}$ & $0.04 \pm 0.00^{\mathrm{b}}$ \\
\hline $20: 1 n-9$ & $0.59 \pm 0.05^{\mathrm{a}}$ & $0.21 \pm 0.08^{\mathrm{b}}$ & $0.26 \pm 0.09^{b}$ & $0.10 \pm 0.01^{\mathrm{c}}$ \\
\hline $20: 1 n-7$ & $0.11 \pm 0.01^{\mathrm{a}}$ & $0.02 \pm 0.01^{\mathrm{b}}$ & $0.03 \pm 0.01^{\mathrm{b}}$ & $0.03 \pm 0.00^{\mathrm{b}}$ \\
\hline $16: 3 n-6$ & $0.09 \pm 0.01^{\mathrm{a}}$ & $0.08 \pm 0.02^{\mathrm{b}}$ & $0.06 \pm 0.04^{\mathrm{b}}$ & $0.02 \pm 0.01^{\mathrm{a}}$ \\
\hline $16: 3 n-4$ & $0.01 \pm 0.00^{\mathrm{a}}$ & $0.08 \pm 0.06^{b}$ & $0.00 \pm 0.00^{\mathrm{a}}$ & $0.01 \pm 0.02^{\mathrm{a}}$ \\
\hline $16: 4 n-3$ & $0.02 \pm 0.00^{\mathrm{a}}$ & $0.10 \pm 0.03^{b}$ & $0.02 \pm 0.01^{\mathrm{a}}$ & $0.00 \pm 0.00^{\mathrm{a}}$ \\
\hline $18: 2 n-6$ & $0.10 \pm 0.01^{\mathrm{a}}$ & $0.70 \pm 0.26^{b}$ & $2.06 \pm 0.52^{\mathrm{c}}$ & $0.03 \pm 0.01^{\mathrm{a}}$ \\
\hline $18: 2 n-4$ & $0.00 \pm 0.00^{\mathrm{a}}$ & $0.01 \pm 0.01^{\mathrm{a}}$ & $0.01 \pm 0.01^{\mathrm{a}}$ & $0.00 \pm 0.00^{\mathrm{a}}$ \\
\hline $18: 3 n-6$ & $0.05 \pm 0.00^{\mathrm{a}}$ & $0.02 \pm 0.01^{\mathrm{b}}$ & $0.02 \pm 0.00^{\mathrm{b}}$ & $0.00 \pm 0.00^{c}$ \\
\hline $18: 3 n-3$ & $0.25 \pm 0.02^{\mathrm{a}}$ & $0.66 \pm 0.23^{b}$ & $1.44 \pm 0.28^{c}$ & $0.03 \pm 0.00^{\mathrm{d}}$ \\
\hline $18: 4 n-3$ & $0.36 \pm 0.03^{a}$ & $0.66 \pm 0.24^{b}$ & $1.70 \pm 0.32^{\mathrm{c}}$ & $0.04 \pm 0.01^{\text {bd }}$ \\
\hline $20: 2 \mathrm{i}$ & $0.12 \pm 0.01^{\mathrm{a}}$ & $0.02 \pm 0.01^{\mathrm{b}}$ & $0.03 \pm 0.01^{\mathrm{b}}$ & $0.02 \pm 0.00^{\mathrm{b}}$ \\
\hline $20: 2 \mathrm{j}$ & $0.03 \pm 0.00^{\mathrm{a}}$ & $0.00 \pm 0.00^{\mathrm{b}}$ & $0.00 \pm 0.00^{\mathrm{b}}$ & $0.00 \pm 0.00^{\mathrm{b}}$ \\
\hline $20: 2 n-6$ & $0.19 \pm 0.01^{\mathrm{a}}$ & $0.18 \pm 0.07^{\mathrm{a}}$ & $0.32 \pm 0.10^{\mathrm{b}}$ & $0.04 \pm 0.00^{c}$ \\
\hline $20: 3 n-6$ & $0.03 \pm 0.00^{\mathrm{a}}$ & $0.02 \pm 0.01^{\mathrm{b}}$ & $0.03 \pm 0.01^{\mathrm{a}}$ & $0.01 \pm 0.00^{\mathrm{b}}$ \\
\hline $20: 4 n-6$ & $0.16 \pm 0.01^{\mathrm{a}}$ & $0.08 \pm 0.03^{b}$ & $0.08 \pm 0.02^{b}$ & $0.04 \pm 0.00^{c}$ \\
\hline $20: 4 n-3$ & $0.12 \pm 0.01^{\mathrm{a}}$ & $0.03 \pm 0.01^{\mathrm{b}}$ & $0.05 \pm 0.01^{\mathrm{b}}$ & $0.02 \pm 0.00^{c}$ \\
\hline $20: 5 n-3$ & $1.41 \pm 0.13^{\mathrm{a}}$ & $0.44 \pm 0.16^{b}$ & $0.25 \pm 0.03^{c}$ & $0.20 \pm 0.02^{\mathrm{c}}$ \\
\hline $21: 4 n-6$ & $0.02 \pm 0.02^{\mathrm{a}}$ & $0.04 \pm 0.02^{\mathrm{b}}$ & $0.05 \pm 0.03^{b}$ & $0.02 \pm 0.00^{\mathrm{a}}$ \\
\hline $21: 5 n-3$ & $0.18 \pm 0.01^{\mathrm{a}}$ & $0.07 \pm 0.05^{\mathrm{b}}$ & $0.09 \pm 0.02^{\mathrm{b}}$ & $0.03 \pm 0.00^{\mathrm{b}}$ \\
\hline $22: 2 \mathrm{i}$ & $0.43 \pm 0.02^{\mathrm{a}}$ & $0.07 \pm 0.03^{b}$ & $0.11 \pm 0.04^{\mathrm{b}}$ & $0.11 \pm 0.01^{\mathrm{b}}$ \\
\hline $22: 2 \mathrm{j}$ & $0.01 \pm 0.01^{\mathrm{a}}$ & $0.01 \pm 0.00^{\mathrm{a}}$ & $0.01 \pm 0.00^{\mathrm{a}}$ & $0.01 \pm 0.00^{\mathrm{a}}$ \\
\hline $22: 4 n-6$ & $0.06 \pm 0.00^{\mathrm{a}}$ & $0.01 \pm 0.00^{\mathrm{b}}$ & $0.01 \pm 0.01^{\mathrm{b}}$ & $0.01 \pm 0.00^{\mathrm{b}}$ \\
\hline $22: 5 n-6$ & $0.05 \pm 0.00^{\mathrm{a}}$ & $0.02 \pm 0.03^{\mathrm{a}}$ & $0.20 \pm 0.04^{\mathrm{b}}$ & $0.01 \pm 0.00^{\mathrm{a}}$ \\
\hline $22: 5 n-3$ & $0.19 \pm 0.01^{\mathrm{a}}$ & $0.02 \pm 0.01^{\mathrm{b}}$ & $0.05 \pm 0.01^{\mathrm{c}}$ & $0.03 \pm 0.00^{\mathrm{b}}$ \\
\hline $22: 6 n-3$ & $1.01 \pm 0.11^{\mathrm{a}}$ & $0.51 \pm 0.20^{\mathrm{b}}$ & $1.66 \pm 0.27^{c}$ & $0.19 \pm 0.02^{\mathrm{b}}$ \\
\hline$\sum$ SAFA & $5.68 \pm 0.48^{\mathrm{a}}$ & $5.78 \pm 1.60^{\mathrm{a}}$ & $6.84 \pm 1.66^{\mathrm{a}}$ & $1.00 \pm 0.22^{\mathrm{b}}$ \\
\hline$\sum$ MUFA & $4.80 \pm 0.40^{\mathrm{a}}$ & $3.58 \pm 1.37^{\mathrm{a}}$ & $5.10 \pm 1.42^{\mathrm{a}}$ & $0.82 \pm 0.07^{\mathrm{b}}$ \\
\hline$\sum n-9$ & $2.07 \pm 0.17^{\mathrm{a}}$ & $1.43 \pm 0.57^{\mathrm{b}}$ & $2.84 \pm 0.81^{\mathrm{a}}$ & $0.41 \pm 0.05^{\mathrm{c}}$ \\
\hline$\sum n-7$ & $2.37 \pm 0.20^{\mathrm{a}}$ & $1.81 \pm 0.65^{\mathrm{a}}$ & $2.07 \pm 0.55^{\mathrm{a}}$ & $0.32 \pm 0.02^{\mathrm{b}}$ \\
\hline$\sum$ PUFA & $4.94 \pm 0.43^{\mathrm{a}}$ & $4.07 \pm 1.45^{\mathrm{a}}$ & $8.29 \pm 1.75^{\mathrm{b}}$ & $0.89 \pm 0.07^{\mathrm{c}}$ \\
\hline$\sum n-6$ & $0.59 \pm 0.05^{\mathrm{a}}$ & $1.06 \pm 0.36^{\mathrm{a}}$ & $2.75 \pm 0.73^{b}$ & $0.14 \pm 0.02^{\mathrm{c}}$ \\
\hline$\sum n-3$ & $3.58 \pm 0.33^{\mathrm{a}}$ & $2.54 \pm 0.92^{\mathrm{a}}$ & $5.29 \pm 0.95^{\mathrm{b}}$ & $0.56 \pm 0.06^{c}$ \\
\hline$\sum$ NMID & $0.59 \pm 0.04^{\mathrm{a}}$ & $0.10 \pm 0.04^{\mathrm{b}}$ & $0.15 \pm 0.06^{\mathrm{b}}$ & $0.14 \pm 0.01^{\mathrm{b}}$ \\
\hline$n-3 / n-6$ & $6.06 \pm 0.09^{\mathrm{a}}$ & $2.38 \pm 0.32^{\mathrm{b}}$ & $1.96 \pm 0.19^{\mathrm{b}}$ & $4.11 \pm 0.66^{\mathrm{c}}$ \\
\hline $22: 6 / 20: 5$ & $0.72 \pm 0.05^{\mathrm{a}}$ & $1.16 \pm 0.09^{\mathrm{b}}$ & $6.64 \pm 0.15^{\mathrm{c}}$ & $0.95 \pm 0.05^{\mathrm{a}}$ \\
\hline $22: 5 / 20: 4$ & $0.31 \pm 0.01^{\mathrm{a}}$ & $0.25 \pm 0.02^{\mathrm{a}}$ & $2.50 \pm 0.11^{\mathrm{b}}$ & $0.25 \pm 0.01^{\mathrm{a}}$ \\
\hline $20: 5 / 20: 4$ & $8.81 \pm 0.28^{\mathrm{a}}$ & $5.50 \pm 0.12^{\mathrm{b}}$ & $3.13 \pm 0.14^{\mathrm{c}}$ & $5.00 \pm 0.15^{\mathrm{b}}$ \\
\hline TOTAL & $16.05 \pm 1.21^{\mathrm{a}}$ & $13.55 \pm 4.21^{\mathrm{a}}$ & $20.42 \pm 4.88^{\mathrm{a}}$ & $2.97 \pm 0.27^{\mathrm{b}}$ \\
\hline
\end{tabular}

Mean values within the same columns with different superscript letters are significantly different $(P<0.05)$.

the experiment, but they increased in size by $26 \mu \mathrm{m}$ at the end of the experiment. The highest survival rate was observed in starved larvae on day $8(66.4 \%)$; however, 3 days after they had been fed larvae died. Diet 1 exhibited a significantly higher survival rate than diet 2 $(P<0.05), 49.0$ and $28.9 \%$ respectively.

\section{Fatty acid composition of the diets}

Table 2 shows the fatty acid composition of the two different experimental diets. The main fatty acids found in the mixed diet (I. galbana, P. lutheri, C. calcitrans and T. suecica; diet 1) were 14:0, 18:2n-6, 18:1n9, 18:3n-3, 18:4n-3, 16:0 and DHA. Total percentages for saturated (SAFA), monounsaturated (MUFA) and polyunsaturated (PUFA) fatty acids were 28.4, 20.1 and $49.6 \%$ respectively. The $n-3: n-6$ ratio was 2.3 .
The main fatty acids found in the single I. galbana diet (diet 2) were 18:4n-3, 18:3n-3, DHA, 14:0, 16:0, 18:0 and 18:2n-6. The PUFAs and SAFAs were higher than in diet 1 , while MUFAs were lower. The $n-3: n-6$ ratio was 4.1 , nearly double that found in diet 1 .

\section{Fatty acid composition of the larvae}

Table 3 shows the main fatty acid content (ng individual $^{-1}$ ) of neutral lipids at the start and end of the experiment in the three treatments. D-shaped larvae (day 1) stored mainly 16:0, 16:1n-7, EPA, 18:1n-9, 14:0 and DHA in the neutral lipid fraction. At the end of the experiment, larvae fed on the I. galbana diet (diet 2) exhibited greater storage of 14:0, 18:1n-9, 18:2n-6 and DHA in neutral lipids than larvae fed on diet 1 , even though the I. galbana diet provided these fatty 

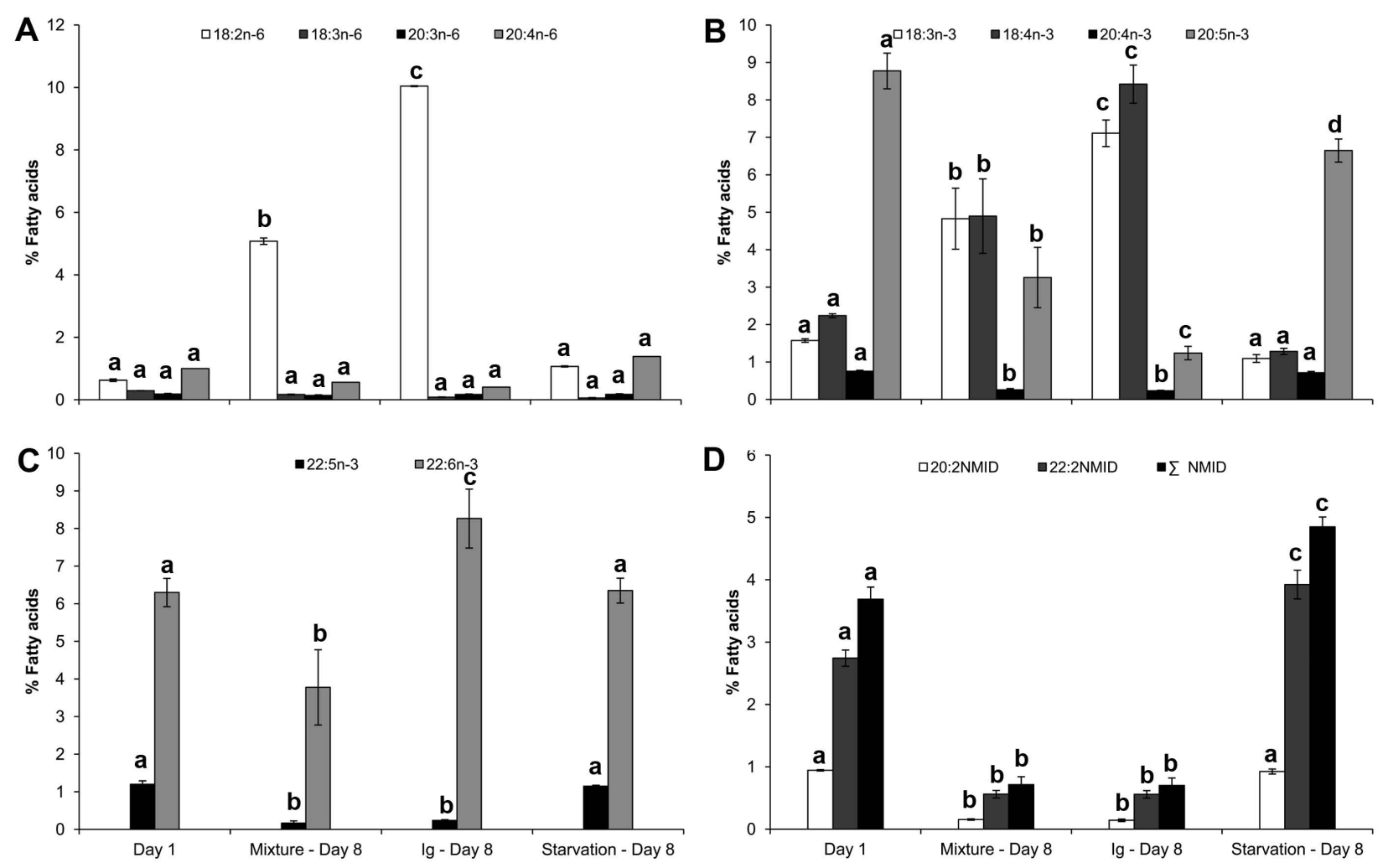

FIG. 1. - Percentage of EPA, DHA, AA and their precursors in neutral lipids of S. marginatus larvae (expressed as mean \pm S.D.). Different superscript letters represent significant differences $(P<0.05)$ of the same fatty acid between treatments.
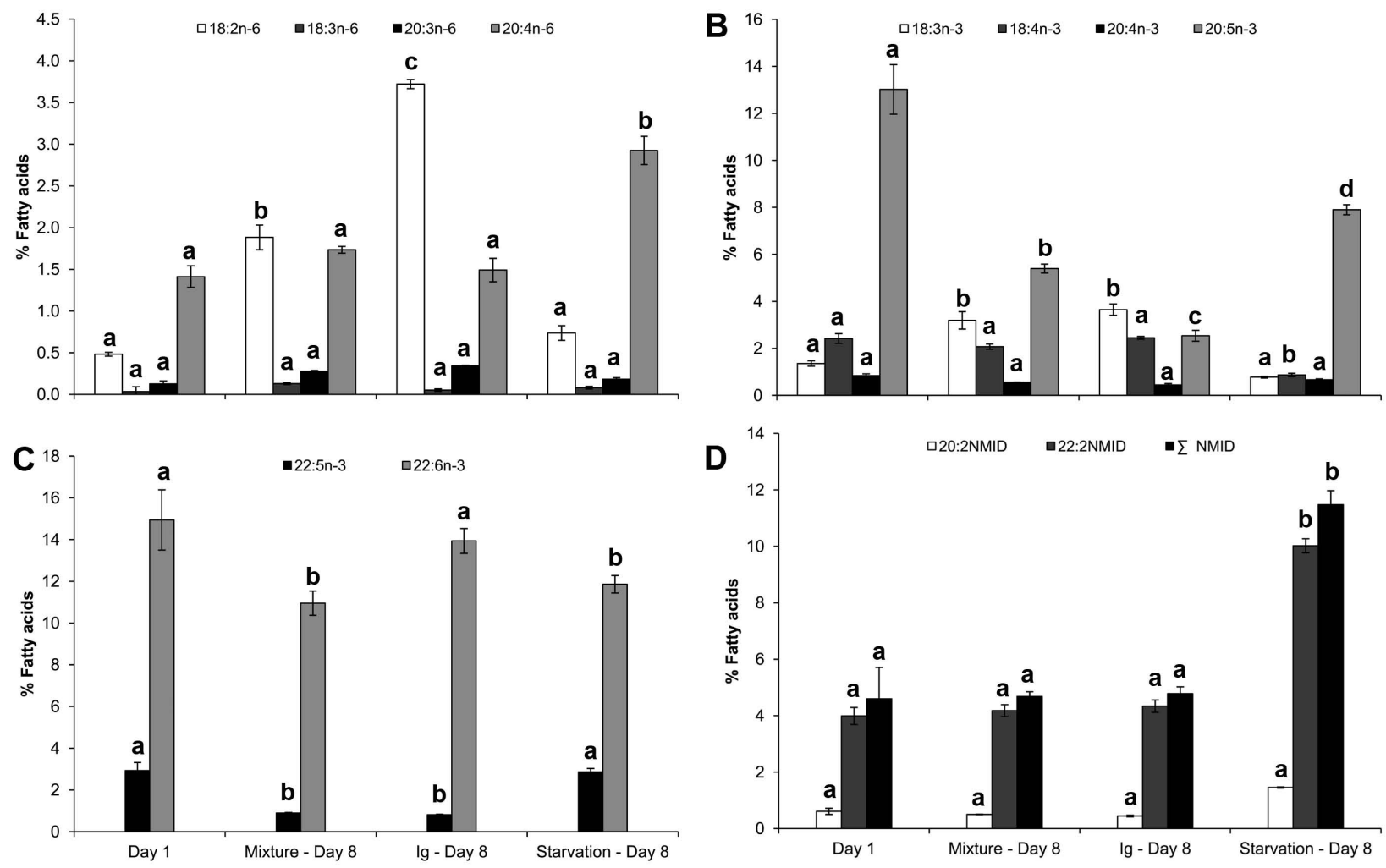

FIG. 2. - Percentage of EPA, DHA, AA and their precursors in polar lipids of S. marginatus larvae (expressed as mean \pm S.D.). Different superscript letters represent significant differences $(P<0.05)$ of the same fatty acid between treatments. 
TABLE 4. - Fatty acid composition of total polar lipids of S. marginatus larvae (expressed as mean \pm S.D. of ng individual-1).

\begin{tabular}{|c|c|c|c|c|}
\hline & Day 1 & Mixture-Day 8 & I. galbana-Day 8 & Starvation-Day 8 \\
\hline 14:0 & $0.032 \pm 0.013^{\mathrm{a}}$ & $0.425 \pm 0.096^{\mathrm{b}}$ & $0.283 \pm 0.128^{b}$ & $0.015 \pm 0.007^{\mathrm{a}}$ \\
\hline $16: 0$ & $0.317 \pm 0.064^{\mathrm{a}}$ & $2.565 \pm 0.530^{\mathrm{b}}$ & $1.573 \pm 0.641^{\mathrm{c}}$ & $0.170 \pm 0.027^{\mathrm{a}}$ \\
\hline 18:0 & $0.108 \pm 0.010^{\mathrm{a}}$ & $1.752 \pm 0.332^{b}$ & $0.950 \pm 0.345^{\mathrm{c}}$ & $0.113 \pm 0.013^{\mathrm{a}}$ \\
\hline $16: 1 n-9$ & $0.003 \pm 0.003^{\mathrm{a}}$ & $0.042 \pm 0.015^{\mathrm{b}}$ & $0.026 \pm 0.017^{\mathrm{b}}$ & $0.005 \pm 0.002^{\mathrm{a}}$ \\
\hline $16: 1 n-7$ & $0.055 \pm 0.016^{\mathrm{a}}$ & $0.301 \pm 0.089^{b}$ & $0.167 \pm 0.081^{\mathrm{c}}$ & $0.021 \pm 0.007^{a}$ \\
\hline $18: 1 n-11$ & $0.000 \pm 0.000^{\mathrm{a}}$ & $0.005 \pm 0.001^{\mathrm{b}}$ & $0.009 \pm 0.001^{\mathrm{b}}$ & $0.000 \pm 0.001^{\mathrm{a}}$ \\
\hline $18: 1 n-9$ & $0.070 \pm 0.018^{\mathrm{a}}$ & $0.817 \pm 0.243^{b}$ & $0.840 \pm 0.403^{b}$ & $0.059 \pm 0.011^{\mathrm{a}}$ \\
\hline $18: 1 n-7$ & $0.038 \pm 0.010^{\mathrm{a}}$ & $0.567 \pm 0.144^{b}$ & $0.297 \pm 0.132^{c}$ & $0.026 \pm 0.005^{\mathrm{a}}$ \\
\hline $20: 1 n-11$ & $0.017 \pm 0.006^{\mathrm{a}}$ & $0.120 \pm 0.037^{b}$ & $0.064 \pm 0.030^{\mathrm{a}}$ & $0.026 \pm 0.004^{\mathrm{a}}$ \\
\hline $20: 1 n-9$ & $0.035 \pm 0.011^{\mathrm{a}}$ & $0.455 \pm 0.120^{\mathrm{b}}$ & $0.328 \pm 0.155^{\mathrm{b}}$ & $0.032 \pm 0.006^{\mathrm{a}}$ \\
\hline $20: 1 n-7$ & $0.003 \pm 0.003^{\mathrm{a}}$ & $0.086 \pm 0.021^{\mathrm{b}}$ & $0.036 \pm 0.017^{\mathrm{c}}$ & $0.012 \pm 0.002^{\mathrm{a}}$ \\
\hline $22: 1 n-9$ & $0.008 \pm 0.007^{\mathrm{a}}$ & $0.000 \pm 0.000^{\mathrm{b}}$ & $0.000 \pm 0.000^{\mathrm{b}}$ & $0.000 \pm 0.000^{\mathrm{b}}$ \\
\hline $16: 3 n-6$ & $0.002 \pm 0.002^{\mathrm{a}}$ & $0.038 \pm 0.017^{\mathrm{b}}$ & $0.018 \pm 0.015^{\mathrm{b}}$ & $0.005 \pm 0.001^{\mathrm{a}}$ \\
\hline $16: 4 n-3$ & $0.000 \pm 0.000^{\mathrm{a}}$ & $0.022 \pm 0.008^{\mathrm{b}}$ & $0.002 \pm 0.003^{\mathrm{a}}$ & $0.000 \pm 0.001^{\mathrm{a}}$ \\
\hline $18: 2 n-6$ & $0.007 \pm 0.002^{\mathrm{a}}$ & $0.289 \pm 0.087^{b}$ & $0.366 \pm 0.162^{b}$ & $0.010 \pm 0.002^{\mathrm{a}}$ \\
\hline $18: 2 n-4$ & $0.000 \pm 0.000^{\mathrm{a}}$ & $0.013 \pm 0.007^{\mathrm{b}}$ & $0.004 \pm 0.003^{a}$ & $0.000 \pm 0.000^{\mathrm{a}}$ \\
\hline $18: 3 n-6$ & $0.001 \pm 0.001^{\mathrm{a}}$ & $0.019 \pm 0.004^{\mathrm{b}}$ & $0.005 \pm 0.003^{\mathrm{a}}$ & $0.001 \pm 0.000^{\mathrm{a}}$ \\
\hline $18: 3 n-3$ & $0.020 \pm 0.005^{\mathrm{a}}$ & $0.492 \pm 0.164^{b}$ & $0.353 \pm 0.141^{\mathrm{b}}$ & $0.010 \pm 0.002^{\mathrm{a}}$ \\
\hline $18: 4 n-3$ & $0.035 \pm 0.007^{a}$ & $0.314 \pm 0.080^{b}$ & $0.241 \pm 0.107^{b}$ & $0.011 \pm 0.002^{\mathrm{a}}$ \\
\hline $20: 2 \mathrm{i}$ & $0.007 \pm 0.003^{\mathrm{a}}$ & $0.042 \pm 0.010^{\mathrm{b}}$ & $0.032 \pm 0.017^{\mathrm{b}}$ & $0.013 \pm 0.002^{\mathrm{a}}$ \\
\hline $20: 2 \mathrm{j}$ & $0.002 \pm 0.003^{\mathrm{a}}$ & $0.033 \pm 0.007^{\mathrm{b}}$ & $0.013 \pm 0.006^{c}$ & $0.006 \pm 0.001^{\mathrm{a}}$ \\
\hline $20: 2 n-6$ & $0.022 \pm 0.007^{\mathrm{a}}$ & $0.479 \pm 0.130^{\mathrm{b}}$ & $0.347 \pm 0.163^{b}$ & $0.018 \pm 0.002^{\mathrm{a}}$ \\
\hline $20: 3 n-6$ & $0.002 \pm 0.002^{\mathrm{a}}$ & $0.042 \pm 0.010^{\mathrm{b}}$ & $0.034 \pm 0.016^{\mathrm{b}}$ & $0.002 \pm 0.000^{\mathrm{a}}$ \\
\hline $20: 4 n-6$ & $0.021 \pm 0.006^{\mathrm{a}}$ & $0.262 \pm 0.060^{\mathrm{b}}$ & $0.151 \pm 0.078^{c}$ & $0.038 \pm 0.005^{\mathrm{a}}$ \\
\hline $20: 4 n-3$ & $0.012 \pm 0.003^{\mathrm{a}}$ & $0.084 \pm 0.020^{\mathrm{b}}$ & $0.042 \pm 0.015^{\mathrm{c}}$ & $0.009 \pm 0.001^{\mathrm{a}}$ \\
\hline $20: 5 n-3$ & $0.191 \pm 0.049^{\mathrm{a}}$ & $0.816 \pm 0.189^{b}$ & $0.244 \pm 0.095^{\mathrm{a}}$ & $0.103 \pm 0.011^{\mathrm{c}}$ \\
\hline $21: 4 n-6$ & $0.003 \pm 0.005^{\mathrm{a}}$ & $0.067 \pm 0.013^{\mathrm{b}}$ & $0.042 \pm 0.009^{c}$ & $0.009 \pm 0.003^{a}$ \\
\hline $21: 5 n-3$ & $0.039 \pm 0.011^{\mathrm{a}}$ & $0.338 \pm 0.061^{\mathrm{b}}$ & $0.184 \pm 0.080^{c}$ & $0.031 \pm 0.003^{\mathrm{a}}$ \\
\hline $22: 2 \mathrm{i}$ & $0.059 \pm 0.022^{\mathrm{a}}$ & $0.581 \pm 0.142^{b}$ & $0.413 \pm 0.199^{b}$ & $0.125 \pm 0.013^{a}$ \\
\hline $22: 2 \mathrm{j}$ & $0.000 \pm 0.000^{\mathrm{a}}$ & $0.051 \pm 0.011^{\mathrm{b}}$ & $0.018 \pm 0.006^{\mathrm{c}}$ & $0.005 \pm 0.000^{\mathrm{a}}$ \\
\hline $22: 4 n-6$ & $0.012 \pm 0.004^{\mathrm{a}}$ & $0.060 \pm 0.013^{\mathrm{b}}$ & $0.038 \pm 0.017^{c}$ & $0.019 \pm 0.002^{\mathrm{a}}$ \\
\hline $22: 5 n-6$ & $0.015 \pm 0.005^{\mathrm{a}}$ & $0.274 \pm 0.053^{b}$ & $0.214 \pm 0.098^{b}$ & $0.014 \pm 0.001^{\mathrm{a}}$ \\
\hline $22: 5 n-3$ & $0.043 \pm 0.013^{\mathrm{a}}$ & $0.136 \pm 0.032^{\mathrm{b}}$ & $0.080 \pm 0.036^{\mathrm{c}}$ & $0.037 \pm 0.003^{\mathrm{a}}$ \\
\hline $22: 6 n-3$ & $0.220 \pm 0.059^{a}$ & $1.661 \pm 0.427^{b}$ & $1.376 \pm 0.632^{b}$ & $0.154 \pm 0.015^{a}$ \\
\hline$\sum$ SAFA & $0.413 \pm 0.037^{\mathrm{a}}$ & $5.092 \pm 1.022^{\mathrm{b}}$ & $3.006 \pm 1.188^{c}$ & $0.318 \pm 0.047^{a}$ \\
\hline$\sum$ MUFA & $0.234 \pm 0.063^{\mathrm{a}}$ & $2.592 \pm 0.700^{\mathrm{b}}$ & $1.838 \pm 0.845^{\mathrm{b}}$ & $0.186 \pm 0.034^{a}$ \\
\hline$\sum n-9$ & $0.116 \pm 0.028^{\mathrm{a}}$ & $1.315 \pm 0.378^{b}$ & $1.193 \pm 0.575^{\mathrm{b}}$ & $0.096 \pm 0.017^{\mathrm{a}}$ \\
\hline$\sum \mathrm{n}-7$ & $0.098 \pm 0.029^{\mathrm{a}}$ & $0.970 \pm 0.256^{\mathrm{b}}$ & $0.514 \pm 0.239^{c}$ & $0.061 \pm 0.014^{\mathrm{a}}$ \\
\hline$\sum$ PUFA & $0.715 \pm 0.200^{\mathrm{a}}$ & $6.187 \pm 1.525^{b}$ & $4.251 \pm 1.898^{c}$ & $0.627 \pm 0.067^{\mathrm{a}}$ \\
\hline$\sum n-4$ & $0.000 \pm 0.000^{\mathrm{a}}$ & $0.018 \pm 0.007^{\mathrm{b}}$ & $0.004 \pm 0.003^{a}$ & $0.000 \pm 0.000^{\mathrm{a}}$ \\
\hline$\sum n-6$ & $0.063 \pm 0.020^{\mathrm{a}}$ & $1.279 \pm 0.320^{\mathrm{b}}$ & $1.075 \pm 0.478^{\mathrm{b}}$ & $0.080 \pm 0.012^{\mathrm{a}}$ \\
\hline$\sum n-3$ & $0.562 \pm 0.148^{a}$ & $3.920 \pm 0.983^{b}$ & $2.542 \pm 1.112^{\mathrm{b}}$ & $0.359 \pm 0.036^{\mathrm{a}}$ \\
\hline$\sum$ NMID & $0.068 \pm 0.028^{\mathrm{a}}$ & $0.707 \pm 0.160^{\mathrm{b}}$ & $0.477 \pm 0.226^{\mathrm{c}}$ & $0.149 \pm 0.016^{\mathrm{d}}$ \\
\hline$n-3 / n-6$ & $9.100 \pm 1.325^{\mathrm{a}}$ & $3.067 \pm 0.086^{\mathrm{b}}$ & $2.376 \pm 0.061^{\mathrm{b}}$ & $4.529 \pm 0.364^{c}$ \\
\hline $22: 6 / 20: 5$ & $10.476 \pm 0.162^{\mathrm{a}}$ & $2.024 \pm 0.104^{b}$ & $5.634 \pm 0.492^{c}$ & $1.495 \pm 0.132^{\mathrm{a}}$ \\
\hline $22: 5 / 20: 4$ & $0.714 \pm 0.002^{\mathrm{a}}$ & $1.046 \pm 0.052^{\mathrm{b}}$ & $1.417 \pm 0.181^{\mathrm{c}}$ & $0.368 \pm 0.011^{\mathrm{d}}$ \\
\hline $20: 5 / 20: 4$ & $9.095 \pm 0.033^{\mathrm{a}}$ & $3.115 \pm 0.127^{\mathrm{b}}$ & $1.616 \pm 0.083^{c}$ & $2.711 \pm 0.009^{b}$ \\
\hline Total FA & $1.456 \pm 0.277^{\mathrm{a}}$ & $15.142 \pm 3.568^{b}$ & $9.843 \pm 4.383^{c}$ & $1.300 \pm 0.170^{\mathrm{a}}$ \\
\hline
\end{tabular}

Mean values within the same columns with different superscript letters are significantly different $(P<0.05)$.

acids in a lower quantity than the mixed diet (diet 1). Conversely, diet 2 led to a higher accumulation of 16:0, $18: 3 n-3$ and $18: 4 n-3$ in larvae compared to those fed with diet 1 , despite the lower fatty acid composition of the diet. Larvae fed diet 2 on day 8 stored less EPA in the neutral fatty acids than those fed diet 1. Larvae fed diet 2 stored more total SAFAs, MUFAs, PUFAs and total fatty acids in neutral lipids at the end of the experiment. Neutral fatty acids stored in the larvae exposed to 8 days of starvation were 16:0, 18:1n-9, 18:0, EPA and DHA. Larvae fed on diet 2 accumulated a similar amount of fatty acids in neutral lipids than those fed diet $1(P>0.05)$. Total fatty acids in neutral lipids were depleted to $2.97 \mathrm{ng}^{\text {individual }}{ }^{-1}$ in starved larvae.

A higher proportion of 18:2n-6 was observed in larvae fed diet $2(P<0.05)$, although no differences were found in the AA proportion (Fig. 1). Diet 2 led to greater storage in larvae of 18:3n-3 and 18:4n-3 than diet 1, whilst EPA was present in a higher proportion in larvae fed diet 1 . Larvae fed diet 1 showed a lower percentage of DHA than those fed on diet $2(P<0.05)$. Non-methylene-interrupted dienoic fatty acids (NMID) were lower in both diets compared to 1 day old larvae $(P<0.05)$. The total NMID percentage was higher in starved larvae compared to the rest of the groups studied $(P<0.05)$.

The SAFAs 14:0, 16:0 and 18:0 were selectively incorporated in the polar lipids of larvae fed on diet 1 , and in higher amounts than in the larvae fed on diet 2 (Table 4). The MUFA 18:1n-9 was not affected by the diet, showing similar amounts in the polar lipids, while 18:1n-7 was higher in larvae fed on diet 1 due 
TABLE 5. - Variations of fatty acids in neutral and polar lipids in 8 day-old larvae with respect to 1 day-old larvae expressed in ng individual ${ }^{-1}$. Variations are calculated from mean values.

\begin{tabular}{|c|c|c|c|c|}
\hline & & & & \\
\hline & Diet 1-Mixture & Diet 2-I. galbana & Diet 1-Mixture & Diet 2-I. galbana \\
\hline 14:0 & 0.852 & 1.336 & 0.394 & 0.251 \\
\hline 16:0 & -0.735 & -0.284 & 2.249 & 1.256 \\
\hline 18:0 & -0.070 & 0.045 & 1.644 & 0.842 \\
\hline 20:0 & -0.005 & -0.008 & 0.017 & 0.008 \\
\hline $22: 0$ & 0.002 & -0.004 & 0.006 & 0.003 \\
\hline $24: 0$ & -0.005 & 0.006 & 0.012 & 0.011 \\
\hline $16: 1 n-9$ & 0.000 & 0.031 & 0.039 & 0.023 \\
\hline $16: 1 n-7$ & -0.440 & -0.274 & 0.246 & 0.112 \\
\hline $18: 1 \mathrm{n}-11$ & 0.057 & 0.001 & 0.005 & 0.009 \\
\hline $18: 1 n-9$ & -0.259 & 1.070 & 0.748 & 0.770 \\
\hline $18: 1 n-7$ & -0.008 & 0.078 & 0.529 & 0.259 \\
\hline $20: 1 \mathrm{n}-11$ & -0.049 & -0.082 & 0.103 & 0.047 \\
\hline $20: 1 n-9$ & -0.383 & -0.331 & 0.419 & 0.292 \\
\hline $20: 1 \mathrm{n}-7$ & -0.082 & -0.080 & 0.036 & 0.030 \\
\hline $16: 3 n-6$ & -0.008 & -0.029 & 0.000 & 0.016 \\
\hline $16: 3 n-4$ & 0.071 & -0.009 & 0.000 & 0.000 \\
\hline $16: 4 n-3$ & 0.075 & -0.006 & 0.022 & 0.002 \\
\hline $18: 2 n-6$ & 0.598 & 1.956 & 0.281 & 0.359 \\
\hline $18: 2 n-4$ & 0.015 & 0.012 & 0.013 & 0.004 \\
\hline $18: 3 n-6$ & -0.025 & -0.030 & 0.019 & 0.005 \\
\hline $18: 3 n-3$ & 0.403 & 1.187 & 0.472 & 0.333 \\
\hline $18: 4 n-3$ & 0.304 & 1.345 & 0.279 & 0.206 \\
\hline $20: 2 \mathrm{i}$ & -0.099 & -0.092 & 0.035 & 0.024 \\
\hline $20: 2 \mathrm{j}$ & -0.030 & -0.029 & 0.031 & 0.011 \\
\hline $20: 2 n-6$ & -0.018 & 0.127 & 0.458 & 0.325 \\
\hline $20: 3 n-6$ & -0.011 & 0.006 & 0.040 & 0.032 \\
\hline $20: 4 n-6$ & -0.084 & -0.078 & 0.241 & 0.130 \\
\hline $20: 4 n-3$ & -0.087 & -0.074 & 0.072 & 0.030 \\
\hline $20: 5 n-3$ & -0.968 & -1.161 & 0.625 & 0.053 \\
\hline $21: 4 n-6$ & 0.021 & 0.028 & 0.064 & 0.040 \\
\hline $21: 5 n-3$ & -0.107 & -0.086 & 0.298 & 0.144 \\
\hline $22: 2 \mathrm{i}$ & -0.358 & -0.318 & 0.522 & 0.354 \\
\hline $22: 2 j$ & -0.004 & -0.006 & 0.051 & 0.018 \\
\hline $22: 4 n-6$ & -0.051 & -0.046 & 0.048 & 0.026 \\
\hline $22: 5 n-6$ & -0.034 & 0.144 & 0.260 & 0.200 \\
\hline $22: 5 n-3$ & -0.169 & -0.144 & 0.093 & 0.037 \\
\hline $22: 6 n-3$ & -0.497 & 0.651 & 1.442 & 1.157 \\
\hline$\sum$ SAFA & 0.100 & 1.164 & 4.679 & 2.593 \\
\hline$\sum$ MUFA & -1.219 & 0.299 & 2.358 & 1.605 \\
\hline$\sum n-9$ & -0.639 & 0.774 & 1.199 & 1.077 \\
\hline$\sum n-7$ & -0.556 & -0.301 & 0.872 & 0.416 \\
\hline$\sum$ PUFA & -0.874 & 3.350 & 5.472 & 3.536 \\
\hline & 0.475 & 2.157 & 1.215 & 1.012 \\
\hline$\sum n-3$ & -1.040 & 1.712 & 3.358 & 1.980 \\
\hline$\sum$ NMID & -0.490 & -0.444 & 0.639 & 0.408 \\
\hline TOTAL & -2.493 & 4.368 & 13.686 & 8.387 \\
\hline
\end{tabular}

to the higher amount provided by diet 1 . The PUFAs 20:4n-6 (AA), EPA and DHA were higher in diet 1. Total fatty acids in polar lipids were significantly higher in diet 1 compared to diet $2(P<0.05)$. In the starved larvae total fatty acids in polar lipids were similar to the amount present in the larvae at the start of the experiment.

The PUFA 18:2n-6 was observed in a higher proportion than in diet 2 (Fig. 2). The percentage of DHA was similar in larvae fed on diet 1 and starved larvae. In contrast, the percentage of AA and EPA was higher in starved larvae compared to larvae fed on diets 1 and 2. The 20:2NMID, 22:2NMID and total NMID were significantly higher in the starved larvae compared to the rest of the groups $(P<0.05)$.

In the neutral lipids of the larvae subjected to the experimental diets, it was observed that the four fatty acids which accounted for the highest net increase were the same: $14: 0,18: 2 n-6,18: 3 n-3$ and 18:4n-3, although their order was different (Table 5). A common pattern was observed in the highest net decreases: the losses were led by EPA in both diets. All the fatty acids in the polar lipids studied increased in the fed larvae, the four fatty acids that decreased most were the same in both diets: 16:0, 18:0, DHA and 18:1n-9. Although the ARA supplied in diet 2 was 7.17-fold lower than in diet 1, its increase in the polar lipids was only 1.85-fold lower compared to larvae fed on diet 1 .

The main fatty acids that were observed to decline in neutral lipids of starved larvae with respect to those of day 1 were 16:0, 16:1n-7, EPA and 18:1n-9 (Table $6)$. Meanwhile, the comparison of starved and fed larvae on day 8 showed that the highest negative dif- 
TABLE 6. - Net loss of fatty acids in neutral and polar lipids in the starved larvae on day 8 with respect to those of day 1 (Starved-day 1 ) and differentials of fatty acid content between starved larvae on day 8 and fed larvae on day 8 (Starv-Diet 1 and Starv-Diet 2) expressed in ng individual $^{-1}$. Variations are calculated from mean values.

\begin{tabular}{|c|c|c|c|c|c|c|}
\hline & Starv-day 1 & $\begin{array}{l}\text { Neutral lipids } \\
\text { Starv-Diet } 1\end{array}$ & Starv-Diet 2 & Starvation & $\begin{array}{l}\text { Polar lipids } \\
\text { Starv-D1 }\end{array}$ & Starv-D2 \\
\hline $14: 0$ & -0.943 & -1.796 & -2.280 & -0.017 & -0.410 & -0.268 \\
\hline $16: 0$ & -2.991 & -2.256 & -2.707 & -0.147 & -2.396 & -1.403 \\
\hline $18: 0$ & -0.582 & -0.512 & -0.627 & 0.005 & -1.639 & -0.838 \\
\hline $20: 0$ & -0.024 & -0.019 & -0.016 & -0.002 & -0.019 & -0.011 \\
\hline $22: 0$ & -0.003 & -0.004 & 0.002 & -0.001 & -0.007 & -0.005 \\
\hline $24: 0$ & -0.007 & -0.002 & -0.013 & 0.000 & -0.012 & -0.011 \\
\hline $16: 1 n-9$ & -0.044 & -0.044 & -0.075 & 0.002 & -0.037 & -0.021 \\
\hline $16: 1 n-7$ & -1.424 & -0.984 & -1.150 & -0.034 & -0.280 & -0.146 \\
\hline $18: 1 n-11$ & 0.001 & -0.056 & 0.000 & 0.000 & -0.005 & -0.008 \\
\hline $18: 1 n-9$ & -1.108 & -0.849 & -2.178 & -0.010 & -0.758 & -0.780 \\
\hline $18: 1 n-7$ & -0.497 & -0.489 & -0.576 & -0.012 & -0.541 & -0.271 \\
\hline $20: 1 n-11$ & -0.116 & -0.067 & -0.035 & 0.009 & -0.094 & -0.037 \\
\hline 20:1n-9 & -0.486 & -0.103 & -0.154 & -0.003 & -0.422 & -0.296 \\
\hline $20: 1 n-7$ & -0.080 & 0.002 & 0.001 & 0.010 & -0.034 & -0.013 \\
\hline $16: 3 n-6$ & -0.067 & -0.059 & -0.038 & 0.002 & 0.000 & 0.000 \\
\hline $16: 3 n-4$ & -0.001 & -0.072 & 0.008 & 0.000 & 0.002 & 0.002 \\
\hline $16: 4 n-3$ & 0.002 & -0.093 & -0.012 & 0.000 & -0.022 & -0.002 \\
\hline $18: 2 n-6$ & -0.018 & -0.667 & -2.024 & 0.003 & -0.279 & -0.356 \\
\hline $18: 2 n-4$ & -0.069 & -0.013 & -0.010 & 0.000 & -0.013 & -0.004 \\
\hline $18: 3 n-6$ & 0.001 & -0.021 & -0.015 & 0.001 & -0.018 & -0.004 \\
\hline $18: 3 n-3$ & -0.045 & -0.623 & -1.407 & -0.010 & -0.482 & -0.342 \\
\hline $18: 4 n-3$ & -0.220 & -0.625 & -1.666 & -0.024 & -0.303 & -0.230 \\
\hline $20: 2 \mathrm{i}$ & -0.321 & 0.004 & -0.003 & 0.005 & -0.030 & -0.019 \\
\hline $20: 2 \mathrm{j}$ & -0.095 & 0.001 & 0.001 & 0.004 & -0.027 & -0.007 \\
\hline $20: 2 n-6$ & -0.029 & -0.135 & -0.279 & -0.004 & -0.461 & -0.329 \\
\hline $20: 3 n-6$ & -0.153 & -0.013 & -0.030 & 0.000 & -0.040 & -0.031 \\
\hline $20: 4 n-6$ & -0.024 & -0.035 & -0.041 & 0.017 & -0.224 & -0.113 \\
\hline $20: 4 n-3$ & -0.119 & -0.013 & -0.026 & -0.004 & -0.076 & -0.034 \\
\hline $20: 5 n-3$ & -0.100 & -0.243 & -0.050 & -0.088 & -0.713 & -0.141 \\
\hline $21: 4 n-6$ & -1.211 & -0.027 & -0.034 & 0.007 & -0.057 & -0.033 \\
\hline $21: 5 n-3$ & -0.006 & -0.035 & -0.056 & -0.008 & -0.306 & -0.152 \\
\hline $22: 2 \mathrm{i}$ & -0.142 & 0.043 & 0.003 & 0.066 & -0.456 & -0.289 \\
\hline $22: 2 j$ & -0.315 & -0.005 & -0.003 & 0.005 & -0.046 & -0.013 \\
\hline $22: 4 n-6$ & -0.008 & 0.006 & 0.001 & 0.007 & -0.041 & -0.019 \\
\hline $22: 5 n-6$ & -0.045 & -0.008 & -0.186 & 0.000 & -0.260 & -0.200 \\
\hline $22: 5 n-3$ & -0.042 & 0.011 & -0.014 & -0.006 & -0.098 & -0.043 \\
\hline $22: 6 n-3$ & -0.158 & -0.326 & -1.474 & -0.066 & -1.508 & -1.223 \\
\hline$\sum$ SAFA & -0.823 & -4.771 & -5.836 & -0.095 & -4.774 & -2.688 \\
\hline$\sum$ MUFA & -4.671 & -2.766 & -4.285 & -0.048 & -2.406 & -1.652 \\
\hline$\sum n-9$ & -3.985 & -1.014 & -2.427 & -0.020 & -1.219 & -1.097 \\
\hline$\sum \mathrm{n}-7$ & -1.653 & -1.491 & -1.746 & -0.037 & -0.909 & -0.453 \\
\hline$\sum$ PUFA & -2.047 & -3.174 & -7.398 & -0.088 & -5.561 & -3.625 \\
\hline$\sum n-6$ & -4.048 & -0.926 & -2.608 & 0.017 & -1.199 & -0.995 \\
\hline$\sum n-3$ & -0.451 & -1.978 & -4.729 & -0.203 & -3.561 & -2.184 \\
\hline$\sum$ NMID & -3.018 & 0.043 & -0.003 & 0.080 & -0.558 & -0.328 \\
\hline TOTAL & -0.447 & -10.582 & -17.444 & -0.156 & -13.842 & -8.543 \\
\hline
\end{tabular}

ferentials in neutral lipids were for SAFAs 16:0 and 14:0 in both diets, followed by MUFAs 16:1n-7 and 18:1n-9 in the mixed diet (diet 1) and by 18:1n-9 and 18:2n-6 in the single diet (diet 2). Although NMID decreased in the neutral lipids of starved larvae during the study, when starved larvae were compared with fed larvae the NMIDs remained unchanged. The highest net losses in polar lipids in starved larvae were found in 16:0, EPA, DHA and 16:1n-7. When starved larvae were compared with fed larvae a similar behaviour was shown in both diets, as 16:0, 18:0, DHA and 18:1n-9 were the fatty acids that showed the highest negative differentials, although the order of importance of 18:0 and DHA varied among diets. NMIDs in polar lipids of starved larvae increased slightly.

\section{DISCUSSION}

Starved larvae reached the best survival rate at day 8 (66\%): larvae were still actively swimming and showed no sign of exhaustion. However, three days after feeding they all died. Previous studies on starvation of $S$. marginatus larvae pointed out that larvae were able to settle at day 10 post-fertilization after 5 days of starvation once they were re-fed (da Costa, unpublished data). This suggests that after 8 days of starvation $S$. marginatus larvae had lost too many energy reserves to survive after feeding was initiated, thus the "point of no return" or PNR was exceeded. We consider that the PNR may be between day 5 and day 8 in this species. Following standard larval development, $S$. marginatus reach the pediveliger stage on day 5 and settlement oc- 
curs on day 8-9 (da Costa and Martinez-Patiño 2009). Therefore, when the larva is deprived of food for 8 days (duration of standard larval development) the stored reserves needed for metamorphosis are exhausted. Moreover, as suggested by Ben Kheder et al. (2010) the food supply is necessary for stimulating the digestive system in early larvae. Ostrea edulis larvae reached the PNR after 7 days of starvation, when the reserves were exhausted (Labarta et al. 1999), while for Ruditapes philippinarum D-shaped larvae the PNR was found to be at 4.5 days (Yan et al. 2009). Despite larger egg sizes and therefore a greater amount of stored reserves (da Costa et al. 2011), S. marginatus larvae need to feed on microalgae to undergo metamorphosis.

Larvae fed on diet 1 exhibited slightly higher growth in length than those fed on diet 2, settling at $287.5 \mu \mathrm{m}$ length, although differences were not significant $(P>0.05)$. Larvae fed on diet 1 selectively incorporated more fatty acids in the polar lipid compartment, thus allocating more energy to growth and incorporating fatty acids in membranes than storing fatty acid reserves in neutral lipids. In contrast, those fed on diet 2 stored more fatty acids in the neutral lipid fraction. Moreover, the survival rate of larvae fed on diet 1 was twice as high as that of larvae fed on diet 2 .

According to Helm and Laing (1987), differences in the development between treatments may not be solely due to the presence or absence of essential components in the diet, but also to the equilibrium between them. Diet 1 provided higher amounts of EPA, while diet 2 supplied more DHA. Accordingly, larvae fed on diet 1 showed a higher EPA content in neutral lipids, while larvae fed on diet 2 mainly stored DHA. Larvae fed on diet 1 incorporated more EPA in polar lipids than the larvae fed on diet 2 due to the higher EPA content in the diet. In larvae fed on diet 1 DHA is transferred to polar lipids, thus resulting in a depletion of DHA in the neutral lipids during larval development. DHA in larval polar lipids showed no differences in the two diets assayed, which means that there is a threshold for its incorporation. This highlights the importance of DHA in polar lipids for $S$. marginatus metamorphosis and that DHA dietary variations are not reflected in DHA incorporated in membranes. In neutral lipids for both diets EPA is used as an energy source, and in polar lipids the higher EPA content in diet 1 resulted in higher EPA enrichment in larval polar lipids. It has been reported that in Placopecten magellanicus larvae DHA was preferentially accumulated and/or retained at the expense of EPA, thus suggesting that DHA plays an important role in sustaining larval development (Pernet et al. 2005). A similar pattern was observed in the polar lipids of Pecten maximus (Marty et al. 1992, Delaunay et al. 1993) and Crassadoma gigantea larvae (Whyte et al. 1992).

The importance of AA in invertebrate species is due to the role it plays in eicosanoids production and the stress response (Howard and Stanley 1999). In this study AA was found in low quantities in I. galbana (diet $2,0.17 \%$ of total fatty acids), similar to the values reported for I. galbana grown at $5^{\circ} \mathrm{C}$ (Farías et al. 2003). However, Delaunay et al. (1993) did not find AA in T-Iso, the tropical strain. The percentage of AA in the mixed diet (diet 1 ) was $1.22 \%$. The accumulation of AA in neutral lipids did not seem to be linked to the diet, as there were no significant differences in AA amounts in neutral lipids of 8-day-old larvae fed on the two diets. Although the AA content in diet 1 is 7.2 -fold higher than in diet 2, in polar lipids of the larvae the difference was lower, as it was only 1.85-fold higher in larvae fed on diet 1 compared to those fed on diet 2. Thus, there is selective incorporation of AA in polar lipids of larvae fed with diet 2 because diet 2 has very little AA and the larvae showed a significant increase in the AA value compared to the value in day-1-larvae. Two explanations can be proposed: 1 . That larvae selectively accumulate AA in polar lipids due to its deficiency in diet 2, as previously suggested by Pernet and Tremblay (2004) for Placopecten magellanicus. These authors observed that larvae fed on a severely deficient AA diet selectively accumulated AA by a factor of three compared to the dietary amount; 2 . That $S$. marginatus larvae are able to synthesize AA by desaturation and elongation from 18:2 n-6. This fatty acid, which is abundant in the diets, transforms into 20:2n-6 by means of elongation. We note that 20:2n-6 did not originate from the diet since it is absent in diet 1 and its value is low in diet $2(0.03 \%)$. The desaturation of $20: 2 n-6$ to $20: 3 n-6$ may occur as $20: 3 n-6$ is also present is very low amounts in both diets; however, a certain increase in polar lipids was observed. Therefore, we suggest that the polar lipids of $S$. marginatus larvae could be able to to synthesize AA. In Mesodesma mactroides De Moreno et al. (1976) observed a marked ability to elongate $18: 2 n-6$ to $20: 2 n-6$, but desaturation to AA was not observed. Similarly, Chu and Greaves (1991) reported elongation of $18: 2 n-6$ to $20: 2 n-6$ in Crassostrea virginica, but they only observed desaturation of 18:2-6 in one oyster. In Crassostrea gigas juveniles Waldock and Holland (1974) demonstrated that oysters can elongate and desaturate fatty acids to EPA and DHA to a small degree, but at a low rate not sufficient for sustaining optimum growth. Therefore, AA synthesis in S. marginatus must be demonstrated in future studies with an adequate experimental design.

Starvation affected both the proportion and amount of fatty acids in neutral and polar lipids. The highest losses of fatty acids in neutral lipids of starved larvae were observed in 16:0, 16:1n-7, EPA and 18:1n-9. Moreover, when the neutral lipids in starved larvae on day 8 were compared with those of fed larvae, the greatest differences were found in 16:0, 14:0 and 18:1n-9. Therefore, SAFAs 16:0 and 14:0 play the role of energy sources and 16:1n-7 and 18:1n-9 are linked with NMID production (see discussion below on NMIDs). Moreover, the proportion of EPA+DHA+AA with respect to the total PUFA in neutral lipids of starved larvae is similar to that found in the larvae on day 1 , and 
is higher than in the fed larvae. This suggests that unfed larvae conserve the relative amount of these three fatty acids in their PUFA in neutral lipids as a reservoir for polar lipids. However, EPA+DHA+AA with respect to the total PUFA in polar lipids on day 8 showed similar values in starved and fed larvae (42-47\%), which might be an adaptation of $S$. marginatus larvae in order to adjust membrane fluidity and permeability.

A few studies have reported increases in NMID in starved larvae and spat (Thompson and Harrison 1992, Caers et al. 1999). In the present work, total NMID percentages were significantly higher in starved larvae at the end of the experiment in both neutral and lipid fractions compared to larvae at day 1 and larvae fed on the two different diets. Therefore, in neutral lipids NMIDs are not affected by the feeding supply or starvation. However, their content in the polar lipid fraction is significantly lower in starved larvae. The pathway of biosynthesis of NMID fatty acids by elongation and $\Delta 5$ desaturation of $16: 1 n-7$ and $18: 1 n-9$ was established by Zhukova (1991). In fed larvae 16:1n-7 and 18:1n-9 are provided by the phytoplankton; however, in the starved larvae these fatty acids come from reserves stored in the egg. The higher content of NMID precursors (i.e. 18:1n7 and 18:1n-9) exhibited in diet 1 does not contribute to a higher NMID content in the neutral and polar lipid fractions. Therefore, $S$. marginatus larvae seem to be genetically driven to biosynthesize NMID during larval development independently of the precursors provided by the diet. Likewise, severe depletion of MUFAs in neutral lipids and a slight depletion in polar lipids was observed in the starved larvae. Therefore, it can be proposed that MUFAs in neutral lipids are the precursors of NMID that will be stored in the neutral lipids and subsequently transferred to the polar lipids. This suggests the importance of NMID fatty acids in S. marginatus larvae subjected to food deprivation. Our observations supported the findings of Paradis and Ackman (1977), who suggested that NMID fatty acids were biochemically inert and somehow resistant to catabolism. Two possible functions were proposed for NMID in the neutral lipids of $S$. marginatus larvae (da Costa et al. 2011). NMID in neutral lipids can be a temporary reservoir for NMID in polar lipids or used as an energy source. We consider the first option more realistic.

In summary, the present study highlights that $S$. marginatus larvae need an external phytoplankton supply to undergo metamorphosis. Moreover, the larvae reached the point of no return by 8 days. In addition, NMIDs and their precursors 16:1n-7 and 18:1n-9 were observed to be very important in starved $S$. marginatus larvae. EPA is used as an energy source in neutral lipids in starved and fed larvae, whilst DHA is selectively accumulated in polar lipids in metamorphosis. SAFAs, especially 16:0, are an important energy source during larval development in $S$. marginatus. This study also suggests the possibility that $18: 2 \mathrm{n}-6$ can be bioconverted to AA. Further nutritional studies on $S$. marginatus larval development are needed to validate these results.

\section{ACKNOWLEDGEMENTS}

We are grateful to the staff of the Centro de Cultivos Marinos de Ribadeo-CIMA (Xunta de Galicia). This research was funded by the project Junta Nacional de Cultivos Marinos JACUMAR (MAPA-CC.AA., Spain): Cultivation of new species of bivalve molluscs of interest in hatcheries.

\section{REFERENCES}

Ben Kheder R., Quéré C., Moal J., Robert R. 2010. Effect of nutrition on Crassostrea gigas larval development and the evolution of physiological indices. Part B: Effects of temporary food deprivation. Aquaculture 308: 174-182.

Caers M., Coutteau P., Sorgeloos P. 1999. Dietary impact of algal and artificial diets, fed at different feeding rations, on the growth and fatty acid composition of Tapes philippinarum (L.) spat. Aquaculture 170: 307-322.

Chu F.-L.E., Greaves J. 1991. Metabolism of palmitic, linoleic, and linolenic acids in adult oysters, Crassostrea virginica. Mar. Biol. 110: 229-236.

da Costa F., Martinez-Patiño D. 2009. Culture potential of the razor clam Solen marginatus (Pennant, 1777). Aquaculture 288: 57-64.

da Costa F., Nóvoa S., Ojea J., Martínez-Patiño D. 2011. Changes in biochemical and fatty acid composition of the razor clam Solen marginatus (Solenidae: Bivalvia) during larval development. Mar. Biol. 158: 1829-1840.

Delaunay F., Marty Y., Moal J., Samain J.F. 1993. The effect of monospecific algal diets on growth and fatty acid composition of Pecten maximus (L.) larvae. J. Exp. Mar. Biol. Ecol. 173: 163-179.

De Moreno J.E.A., De Moreno V.J., Brenner R.R. 1976. Lipid metabolism of the yellow clam, Mesodesma mactroides: II: polyunsaturated fatty acid metabolism. Lipids 11: 561-566.

Farías A., Bell J.G., Uriarte I., Sargent J.R. 2003. Polyunsaturated fatty acids in total lipid and phospholipids of chilean scallop Argopecten purpuratus (L.) larvae: effects of diet and temperature. Aquaculture 228: 289-305.

Helm M.M., Laing I. 1987. Preliminary observations on the nutritional value of "Tahiti Isochrysis" to bivalve larvae. Aquaculture 62: 281-288.

Helm M.M., Bourne N., Lovatelli A. (Comp./Ed.) (2004). Hatchery culture of bivalves. A practical manual. FAO Fisheries Technical Paper. No. 471. Rome, 177 pp.

Hendriks I.E., van Duren L.A., Herman P.M.J. 2003. Effect of dietary polyunsaturated fatty acids on reproductive output and larval growth of bivalves. J. Exp. Mar. Biol. Ecol. 296: 199-213.

Howard R.W., Stanley D.W. 1999. The tie that binds: Eicosanoids in invertebrate biology. Ann. Entomol. Soc. Am. 92: 880-890.

Labarta U., Fernandez-Reiriz M.J., Perez-Camacho A. 1999. Larvae of Ostrea edulis (L.) during starvation: growth, energy and biochemical substrates. Hydrobiologia 405: 125-131.

Laing I., Child A.R., Janke A. 1990. Nutritional value of dried algae diets for larvae of Manila clam (Tapes philippinarum). J. Mar. Biol. Ass. U. K. 70: 1-12.

Langdon C.J., Waldock M.J. 1981. The effect of algal and artificial diets on the growth and fatty acid composition of Crassostrea gigas spat. J. Mar. Biol. Ass. U. K. 61: 431-448.

Marshall R., McKinley S., Pearce C.M. 2010. Effects of nutrition on larval growth and survival in bivalves. Rev. Aquacult. 2: 33-55.

Marty Y., Delaunay F., Moal J., Samain J.F. 1992. Changes in the fatty acid composition of Pecten maximus (L.) during larval development. J. Exp. Mar. Biol. Ecol. 163: 221-234.

Metcalfe L.D., Schmitz A.A. 1961. The rapid preparation of fatty acid esters for gas chromatography analysis. Anal. Chem. 33: 363-364.

Ockelmann K.W. 1965. Developmental types in marine bivalves and their distribution along the Atlantic coasts of Europe. Proccedings of the First European Malacological Congress. London, pp. 25-53.

Palacios E., Racotta I.S., Kraffe E., Marty Y., Moal J., Samain 
J.F. 2005. Lipid composition of the giant lion's-paw scallop (Nodipecten subnodosus) in relation to gametogenesis: I. Fatty acids. Aquaculture 250: 270-282.

Paradis M., Ackman R. 1977. Potential for employing the distribution of anomalous non-methylene-interrupted dienoic fatty acids in several marine invertebrates as part of food web studies. Lipids 12: 170-176.

Pernet F., Bricelj V.M., Parrish C.C. 2005. Effect of varying dietary levels of omega 6 polyunsaturated fatty acids during the early ontogeny of the sea scallop, Placopecten magellanicus. J. Exp. Mar. Biol. Ecol. 327: 115-133.

Pernet F., Tremblay R. 2004. Effect of varying levels of dietary essential fatty acid during early ontogeny of the sea scallop Placopecten magellanicus. J. Exp. Mar. Biol. Ecol. 310: 73-86.

Sokal R.R., Rohlf F.J. 1995. Biometry. The principleas and practice of statistics in biological research. Third edition edn. Freeman, New York, 358 pp.

Soudant P., Marty Y., Moal J., Samain J.F. 1995. Separation of major polar lipids in Pecten maximus by high-performance liquid chromatography and subsequent determination of their fatty acids using gas chromatography. J. Chromatogr. B-Biomed. Appl. 673: 15-26.

Thompson P.A., Harrison P.J. 1992. Effects of monospecific algal diets of varying biochemical composition on the growth and survival of Pacific oyster (Crassostrea gigas) larvae. Mar. Biol. 113: 645-654.

Waldock M.J., Holland, D.L. 1984. Fatty acid metabolism in young oysters, Crassostrea gigas: polyunsaturated fatty acids. Lipids 19: 332-336.

Webb K.I., Chu F.E. 1982. Phytoplankton as a food source for bivalve larvae. In: Pruder G.D., Langdon C., Conklin D. (eds.), Proceedings of the second international conference on aquaculture nutrition; biochemical and physiological approaches to shellfish nutrition. Louisiana State University, Baton Rouge, pp. $272-290$.

Whyte J.N.C., Bourne N., Ginther N.G., Hodgson C.A. 1992. Compositional changes in the larva to juvenile development of the scallop Crassadoma gigantea (Gray). J. Exp. Mar. Biol. Ecol. 163: 13-29.

Yan X., Zhang Y., Huo Z., Yang F., Zhang G. 2009. Effects of starvation on larval growth, survival, and metamorphosis of Manila clam Ruditapes philippinarum. Acta Ecol. Sinica 29: 327-334.

Zhukova N.V. 1991. The pathway of the biosynthesis of nonmethylene-interrupted dienoic fatty acids in molluscs. Comp. Biochem. Phys. B. 100: 801-804.

Scient. ed.: M. Ramón.

Received July 28, 2011. Accepted March 7, 2012.

Published online July 18, 2012. 